\title{
Design, Fabrication, and Swimming Performance of a Free-Swimming Tuna-Mimetic Robot
}

\author{
Sayyed Farideddin Masoomi, ${ }^{1}$ Axel Haunholter, ${ }^{2}$ Dominic Merz, ${ }^{3}$ \\ Stefanie Gutschmidt, ${ }^{1}$ XiaoQi Chen, ${ }^{1}$ and Mathieu Sellier ${ }^{1}$ \\ ${ }^{1}$ Department of Mechanical Engineering, University of Canterbury, Private Bag 4800, Christchurch 8041, New Zealand \\ ${ }^{2}$ Department of Applied Physics, Munich University of Applied Sciences, 80335 Munich, Germany \\ ${ }^{3}$ Department of Mechanical and Process Engineering, Darmstadt University of Technology, 64287 Darmstadt, Germany
}

Correspondence should be addressed to Sayyed Farideddin Masoomi; sayyed.masoomi@gmail.com

Received 14 October 2013; Accepted 1 February 2014; Published 26 May 2014

Academic Editor: Yangmin Li

Copyright (C) 2014 Sayyed Farideddin Masoomi et al. This is an open access article distributed under the Creative Commons Attribution License, which permits unrestricted use, distribution, and reproduction in any medium, provided the original work is properly cited.

\begin{abstract}
High efficiency in cruising is a determining factor in developing tuna-mimetic robots. So far, a number of tuna-like robots have been made. Nevertheless, the University of Canterbury has developed its own tuna-like robot called UC-Ika 1 to investigate and to accordingly improve the swimming performance of the biomimetic swimming robots. In order to do so, the propulsion system of a tuna with respect to its thrust and resistive forces is studied. Following that, the fish robot is designed and fabricated considering the tuna propulsion system. The robot is then tested several times to investigate its swimming performance. Comparison of the speed and efficiency of UC-Ika 1 with those of other tuna-like robots shows a promising improvement of cruising performance of UC-Ika 1.
\end{abstract}

\section{Introduction}

The majority of underwater tasks such as monitoring of sea cable and pipelines or pollution search demand a robot with navigation capabilities for a long period of time [1]. For this purpose, biomimetic swimming robots are the most suitable ones due to their fast, very efficient, and highly maneuverable performance [2].

The first biomimetic swimming robot, RoboTuna, was built at MIT in 1994 [3]. In 1997, Vorticity Control Unmanned Undersea Vehicle (VCUUV) was developed based on RoboTuna with some improvement and more capabilities such as avoiding obstacles and having up-down motion [4]. Since then, a number of fish robots with more capabilities are developed [5-8]. The more detailed state of art in robotic fish is presented in [9].

Among the existing fish robots, several robots like RoboTuna and VCUUV are inspired by tuna which is a pelagic fish whose locomotion is highly efficient [10] (bear in mind that Bandyopadhyay [11] believes that the efficiency of biomimetic swimming robot is not higher than screw propeller robots but animals do show superior manoeuvrability in swimming). However, the efficiency of locomotion of tuna-mimetic robots is not thoroughly investigated. Hence, the authors have developed a fish robot that is inspired by a tuna in order to study its cruising efficiency. This paper presents the steps of developing the tuna-mimetic robot in brief including design, modeling, and fabrication and also discusses the swimming performance of the robot in detail.

The paper is organized as follows. Next section focuses on the design criteria of the fish robot. In Section 3, the robot design is introduced. In Section 4, the fabrication process is the center of attention including how the fish is fabricated with which material. Section 5 analyzes the swimming performance of the robot. And finally, the paper is concluded.

\section{Physic Criteria of Tuna Design}

The dynamic behavior of the fish robot is influenced by two main forces: hydrostatic and hydrodynamic forces. 
Hydrostatic forces are more essential for depth control while hydrodynamic ones are used for swimming. However, to facilitate the swimming model with minimum energy dissipation, hydrodynamic forces need to be produced with respect to several factors. These factors are introduced as optimal swimming factors.

2.1. Hydrostatic Forces. Hydrostatic forces such as weight and buoyancy play crucial roles in the stability of fishes. The weight, $W$, is defined as the mass multiplied by the gravitational constant, $M_{f} g$. On the other hand, the buoyancy, $B$, is defined by Archimedes' law as the displaced mass of water multiplied by the gravitational constant, $\rho_{w} V_{f} g$, where $V_{f}$ is the fish volume and $\rho_{w}$ is the density of water. In order to keep the position of the robot stable under water, $W$ and $B$ need to be equal. Additionally, the centers of mass and buoyancy must be vertically aligned while the center of buoyancy should be above that of the weight. This assures the attitude stability of the robot.

2.2. Hydrodynamic Forces. Hydrodynamic forces such as resistive and thrust forces vary from fish to fish. For a tunalike robot, the main resistive force is associated with the pressure drag while the main thrust force is associated with the lift force [12]. Accordingly, the pressure drag and lift forces need to be decreased and increased, respectively, in order to have an efficient swimming.

The pressure drag is the result of the pressure gradient along the body. In order to decrease this drag, the shape of the animal is a determining factor. The best overall shape of swimming animals is to have streamlined bodies with the diameter of the thickest part, $d$, and fish length, $l$. Streamlined bodies with $d / l$ between 0.18 and 0.28 produce less than $10 \%$ of the minimum possible drag [13].

During the motion, tuna fishes generate lift forces through shedding vortices around the tips of their caudal fin. Larger vortices provide greater thrust force. To enlarge these vortices, several factors are contributing. Initially, the caudal fin needs to be lunate-shaped with high aspect ratio (shown in Figure 1). Moreover, the body and the caudal fin should have undulatory motion. Tuna fishes generate a traveling wave through their tail peduncle at greater speeds than swimming speed. This type of motion is called undulation [10]. Undulatory motion requires the caudal fin to change its direction once it reaches its maximum heave. This also helps the fish to create greater vortices.

Note that during undulation the fluid around the fish is pushed and pulled laterally. These accelerations and decelerations of the fluid result in escalation of energy dissipation and reduction of swimming efficiency. Since the tuna fish is an efficient swimmer and its undulation is initiated in its tail peduncle, the joint between the caudal fin and the tail peduncle is as narrow as possible to reduce this energy dissipation. In other words, the smaller surface of the tail peduncle helps tuna to move smaller volume of water laterally. This saves the energy of tuna during cruising.

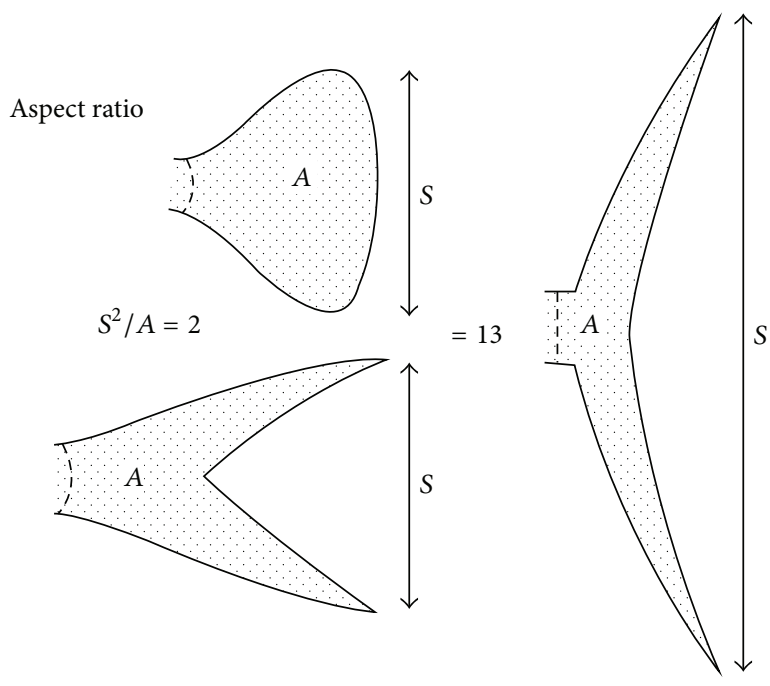

Figure 1: Caudal fin shapes [13].

2.3. Optimal Swimming Factors. While optimized design regarding the shape of the body and the caudal fin can enhance the swimming performance of a fish robot, there exist other decisive factors of designing an efficient swimming robot. Two main criteria are taken into account in this paper: Strouhal number and Froude efficiency.

The Strouhal number is a factor that shows the structure of the vortices made through the body undulation of fishes. The Strouhal number, St, is a dimensionless parameter. It represents the ratio of unsteady to inertial forces and is defined as

$$
\text { St }=2 \frac{f h}{\overline{\dot{x}}},
$$

where $f$ is the frequency of the body undulation, $h$ is the heave of the caudal fin, and $\overline{\dot{x}}$ is the average cruising velocity of the fish. If $0.25<\mathrm{St}<0.4$, the vortices behind the caudal fin produce maximum thrust. Note that the Strouhal number is applicable for fishes whose swimming is through their body and caudal fins [14].

The Froude efficiency is another important factor to evaluate the swimming behavior of fishes. This factor relates the useful power used for propulsion to total kinetic energy of the fish which is the mean rate of transferred momentum to the wake around the fish. Froude efficiency is defined by

$$
\eta=\frac{\overline{F_{C x} \dot{x}}}{P_{\text {total }}},
$$

where $\overline{F_{C x}}$ is the thrust and $\overline{\dot{x}}$ is the mean velocity of the fish. $P_{\text {total }}$ is the total kinetic energy of the fish [15]. In this paper, $P_{\text {total }}$ is obtained through the following expression:

$$
P_{\text {total }}=\overline{F_{C x} \dot{x}}+\overline{F_{C y} \dot{y}},
$$

where $\overline{F_{C y}}$ is the force to generate vortex wake and $\overline{\dot{y}}$ is the mean lateral speed of the caudal fin. Derivations of $\overline{F_{C x}}$ and $\overline{F_{C y}}$ are presented in [16]. A fish could be up to $90 \%$ efficient while a screw propeller fish robot is at most $50 \%$ efficient [17]. 


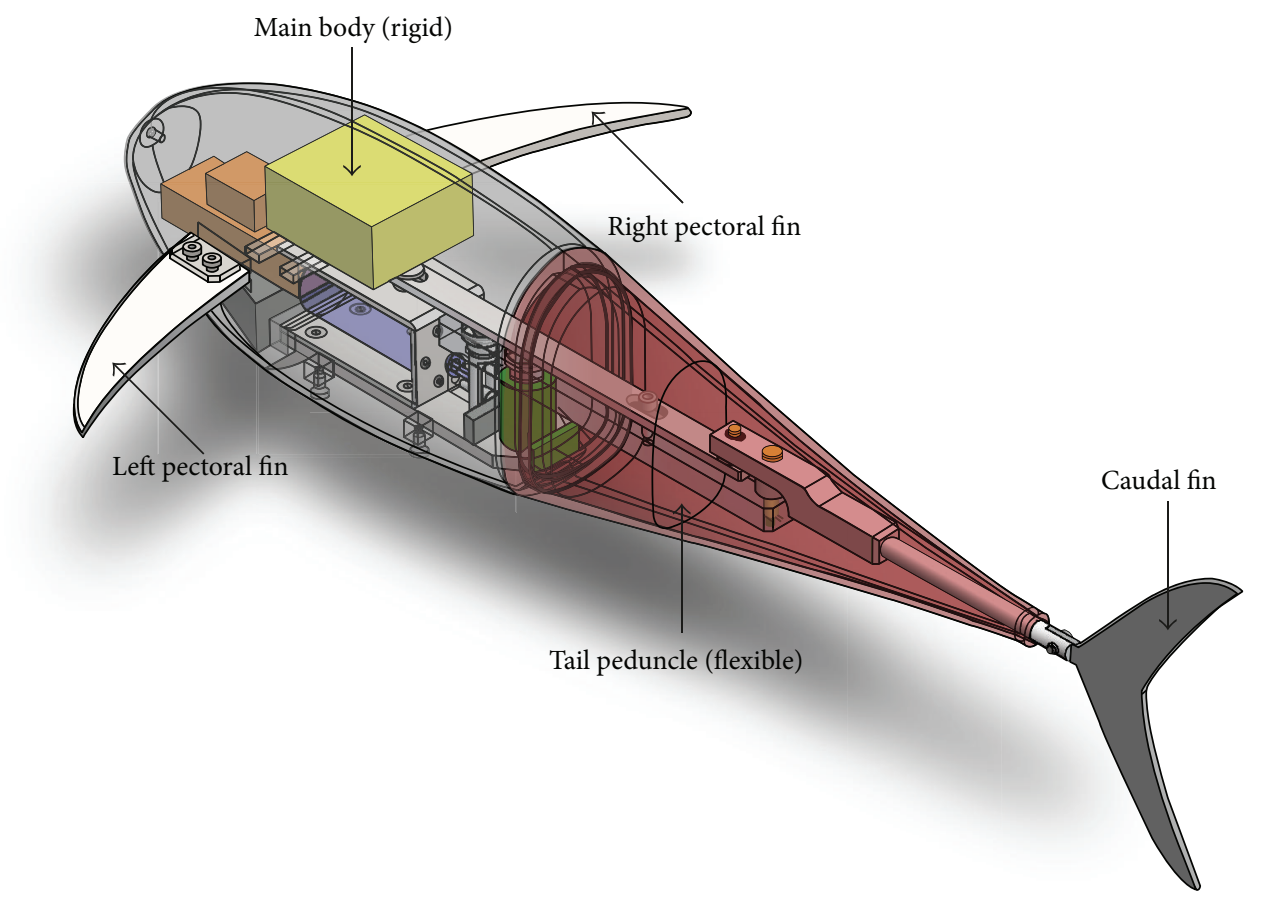

FIgURE 2: The CAD design of UC-Ika 1.

\section{Prototype Design}

Figure 2 illustrates the tuna-like robot designed at the University of Canterbury. Its name, UC-Ika 1, originates from the Maori word for fish which is Ika.

The robot consists of two main parts: the main body and the tail. The main body is designed as a rigid part and contains all stationary components such as batteries, microcontroller, and DC motor. The pectoral fins are fixed to the body and, in this prototype, are rigid as well. The tail includes a flexible tail peduncle and a rigid caudal fin. Inside the tail peduncle, the undulation actuation mechanism is located. The mechanism connects the DC motor with the caudal fin; see Figure 2.

The design of UC-Ika 1 allows investigating all aforementioned physic criteria which are necessary for a stable and efficient cruising motion of the robot. UC-Ika 1 is neutrally buoyant with an approximate weight of $4 \mathrm{~kg}$ while the center of buoyancy is above that of the mass (Figure 3).

Figure 3 also shows that the overall shape of UC-Ika 1 is streamlined with $d$ and $l$ of $147 \mathrm{~mm}$ and $610 \mathrm{~mm}$, respectively. Accordingly, the body shape of the robot has $d / l$ equal to 0.24 which is within the optimal range for streamlined bodies, $0.18-0.28$.

Moreover, in order to have efficient cruising, the tail peduncle skin is designed to be flexible. The tail peduncle at the connection to the caudal fin is narrow with peripheral of $64 \mathrm{~mm}$. The caudal fin is designed to be lunate-shaped with aspect ratio, $S^{2} / A$, of 6.4 , where $S$ is $170 \mathrm{~mm}$ and $A$ is equal to $4500 \mathrm{~mm}^{2}$.

The undulation of the tail is determined by the kinematic mechanism illustrated in Figure 4. This mechanism has

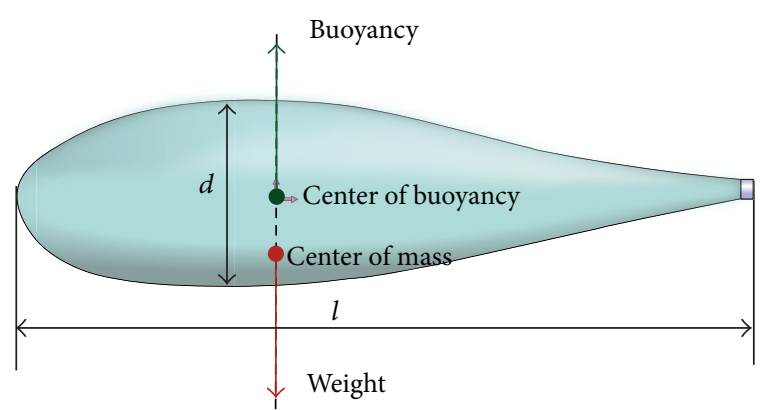

FIgURE 3: The overall body shape of UC-Ika 1.

distinguishing features. First of all, the mechanism is actuated by one DC motor. This allows assembling of the motor inside the main body close to the center of mass in order to decrease the weight of the tail peduncle. When the tail peduncle is light, the robot swinging is controllable due to the small mass moment of inertia.

The second distinguishing feature is the passive control of the third link. In other words, $\theta_{3}$ in Figure 4 is dependent on the motion of the rest of the link system and is determined by $\theta_{1}$. Accordingly, less degrees of freedom need to be controlled.

The third important feature of the mechanism is owing to its suitability for mimicking the real tuna cruising. The existing tuna-mimetic robots have swimming mode of carangiforms like mackerel. Carangiforms undulate from the last third of their body while the lift-based propulsion system of thunniforms like tuna is confined to the very last part of their body near to the caudal fin [10]. Thus the tail mechanism 


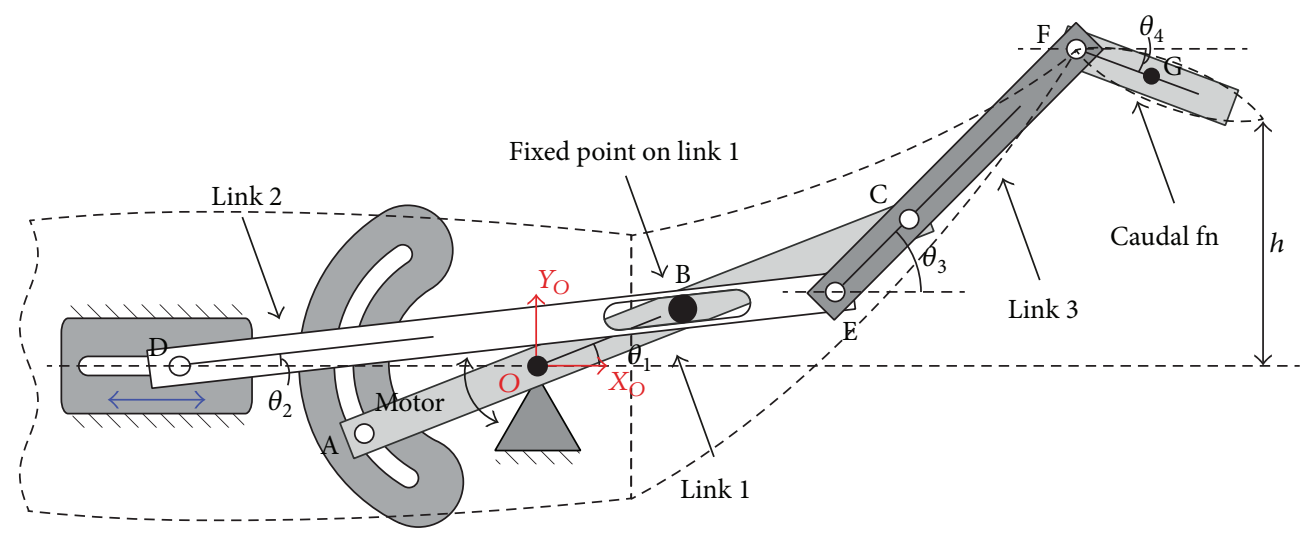

FIgURE 4: The link mechanism of the tail peduncle.

avoids excessive motion of the tail peduncle near to the rigid body and its associated energy dissipation.

Considering the kinematics of the tail design, Figure 4, $\theta_{1}$ amplitudes of 7 degree cause a heave of $17 \mathrm{~mm}$ for point C. On the other hand, the motor oscillation of 14 degree of $\theta_{3}$ yields a heave of $56 \mathrm{~mm}$ for point $\mathrm{F}$. The short heave for point $\mathrm{C}$ and long one for point $\mathrm{F}$, while the tail mechanism is not optimized, are a quite satisfactory result for a tuna-like undulation.

\section{Fabrication and Assembly}

For fabrication of the complex shape of the rigid main body, a rapid prototyping method called fused deposition modeling (FDM) is applied, which is a 3D-printing technology directly using the CAD model. Then the design is fabricated layer by layer using two different melted materials as the base and support materials. The base material, Acrylnitril-ButadienStyrol-Copolymerisat (ABS), is in fact the actual material of the fabrication. After 3D-printing, the support material is resolved and removed from the part in a $70^{\circ} \mathrm{C}$ hot alkaline bath (Figure 5) [18]. In order to make the main body waterproof, it is painted with epoxy resin to avoid passing of water through that over time as the body is slightly porous.

The flexible tail peduncle is produced from polydimethylsiloxane (PDMS) silicone Sylgard 184 which is durable, tensile, and resistant against water and most solvents [19]. The silicone is made up of two components. These two components need to be combined and poured into a mold. The mold of the tail peduncle is also fabricated with the 3Dprinter similar to rigid body fabrication. The solidifying of the tail takes approximately 72 hours. Figure 6 illustrates casting of the flexible tail.

The other components of the fish robot such as pectoral fins, actuation mechanism, and the caudal fin are fabricated with commonly known fabrication machines. Note that the actuation mechanism and pectoral fins are made up of steel and aluminum while the caudal fin is made of a $5 \mathrm{~mm}$ polymethyl-methacrylate (perspex) sheet.

To control the $12 \mathrm{~V}$ DC gear head motor of the fish, an open loop controller is designed. The controller is coded into

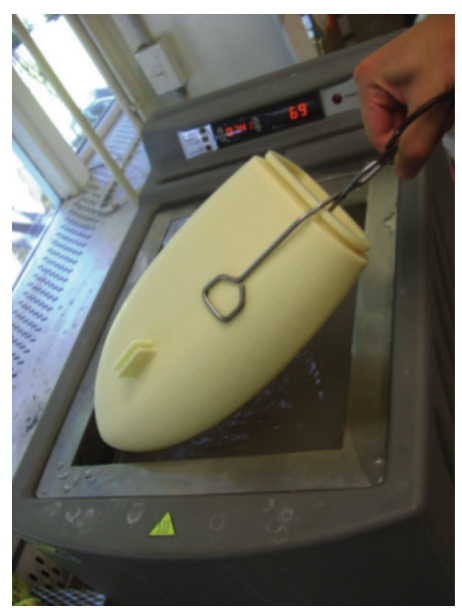

FIGURE 5: Dissolving support material.

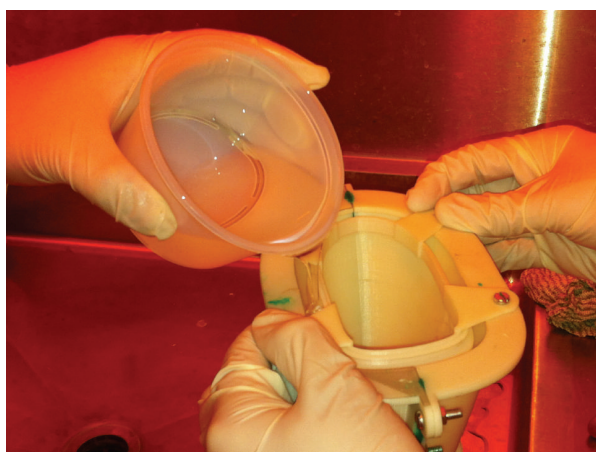

Figure 6: Casting of tail peduncle.

an Arduino Uno microcontroller which could communicate with any Bluetooth device like computers and smartphones using a Bluetooth connector.

Figure 7 shows the final prototype of the fish robot, UC-Ika 1.

\section{Fish Performance}

UC-Ika 1 is a signle gaited robot and is only capable of cruising motion with its tail peduncle and caudal fin. When the caudal 


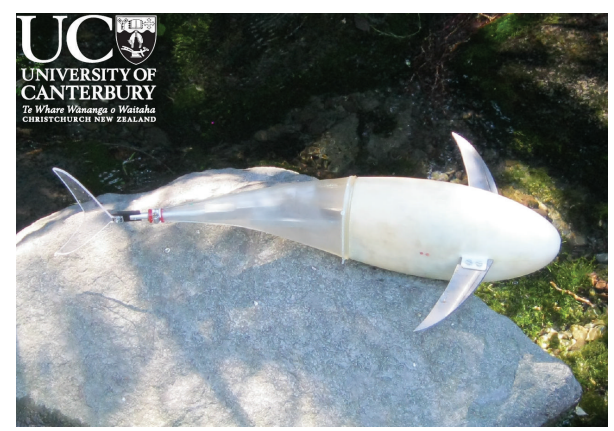

FIGURE 7: First prototype developed at the University of Canterbury, UC-Ika 1.

TABLE 1: Time to swim 1.5 meter by the fish robot.

\begin{tabular}{lcc}
\hline \multirow{2}{*}{ Test } & \multicolumn{2}{c}{ Swimming time (s) } \\
& Fixed joint & Flexible joint \\
\hline 1 & 7.0 & 4.7 \\
2 & 6.8 & 5.5 \\
3 & 7.4 & 5.5 \\
4 & 6.8 & 5.0 \\
5 & 7.0 & 5.2 \\
\hline
\end{tabular}

fin changes its direction at its maximum heave, larger vortices are created which assures a faster performance of the robot. In order to confirm this theory, UC-Ika 1 is tested with two different tail designs: with a fixed joint of the caudal fin shown in Figure 8(a) and a flexible one presented in Figure 8(b). For a fixed joint, the caudal fin has the same orientation of the tail peduncle, while the flexible joint, made by a piece of rubber, causes the caudal fin to change its direction at its maximum heave.

Note that VCUUV is operated with hydraulic power unit and RoboTuna is a carriage mounted robot with external power supply [9] while UC-Ika 1 is actuated with only one internally fixed $12 \mathrm{~V} \mathrm{DC}$ motor. Therefore UC-Ika 1 does not need external support and employs an electric motor which is preferred for medium-sized robots. For further information regarding actuators, the reader is referred to [20].

Multiple tests for both aforementioned caudal fin designs are performed in a water tank of $4 \mathrm{~m}$ length. Table 1 shows the result for each test.

The results reveal a cruising speed of $0.21 \mathrm{~m} / \mathrm{s}$ and $0.29 \mathrm{~m} / \mathrm{s}$ for UC-Ika 1 with fixed and flexible joints, respectively (the results obtained from several tests of the robot are completed by the motion analysis of the video of cruising motion of UC-Ika 1). Fish speeds are commonly measured and compared with respect to their body lengths. Accordingly, the speed of UC-Ika 1 is 0.33 body lengths per second $(\mathrm{BL} / \mathrm{s})$ for the fixed joint and $0.44 \mathrm{BL} / \mathrm{s}$ for the flexible design. These results are comparable with the existing tunalike robots. Figure 9 compares the two tuna-mimetic robots, VCUUV and RoboTuna, to UC-Ika 1 with both fixed and flexible joints.

The optimality of the robot swimming with the flexible joint is also investigated through the Strouhal number and
TABLE 2: Swimming parameters of UC-Ika 1.

\begin{tabular}{lc}
\hline Parameter & Value \\
\hline Undulation frequency & $f=1.5 \mathrm{HZ}$ \\
Heave & $h=0.07 \mathrm{~m}$ \\
Mean forward velocity & $\overline{\dot{x}}=0.29 \mathrm{~m} / \mathrm{s}$ \\
Mean lateral velocity & $\overline{\dot{y}}=0.07 \mathrm{~m} / \mathrm{s}$ \\
Mean thrust & $\overline{F_{C x}}=0.23 \mathrm{~N}$ \\
Mean lateral force & $\overline{F_{C y}}=0.26 \mathrm{~N}$ \\
\hline
\end{tabular}

Froude efficiency. In order to calculate these two quantities, swimming parameters of UC-Ika 1 are primarily obtained from the experiment. Those parameters are shown in Table 2.

It should be noted that $\overline{F_{C x}}$ and $\overline{F_{C y}}$ used in Froude efficiency must be obtained when the robot is in cruising. In cruising mode, the average of the propulsive forces is equal to the average of the resistive forces. Accordingly, $\overline{F_{D x}}$ and $\overline{F_{D y}}$ are calculated with speed of the robot in cruising mode and replaced by $\overline{F_{C x}}$ and $\overline{F_{C y}}$ (e.g., $\overline{F_{C x}}=\overline{F_{D y}}=(1 / 2) C_{D x} \rho S_{x} \overline{\dot{x}}^{2}=$ $0.23 \mathrm{~N})$.

Substituting $f, h$, and $\overline{\dot{x}}$ into (1) yields $S t=0.72$. This value of St demonstrates that the vortices produced by the fish robot are not completely ideal. However, Froude efficiency of UCIka 1 is approximately high. Substituting the values of $\overline{F_{C x}}$, $\overline{F_{C y}}, \overline{\dot{x}}$, and $\overline{\dot{y}}$ into (2) and (3), the Froude efficiency of the fish robot is obtained to be equal to $78 \%$.

The result reveals a promising performance, since several hydrodynamic aspects of tuna fish are not yet taken into account. For instance, the caudal fin does not have streamlined shape. Moreover, the pectoral fins for tuna fish are static and oriented inwards to their bodies while for UC-Ika 1 the pectoral fins are fixed outwards. Besides, the kinematic tail mechanism has not yet been optimized. Future optimized designs are predicted to have higher efficiencies, greater than $83 \%$.

\section{Conclusion}

In order to design a stable, fast, and efficient tuna-like robot, the physic criteria of a tuna propulsion need to be taken into account. Accordingly, the robot needs to be neutral buoyant. The robot requires a streamlined overall shape. The tail needs to be flexible for undulatory motion to provide a large heave for lunate-shaped caudal fin. And finally, the tail peduncle must be narrow near the flexible joint of the caudal fin. UCIka 1 is designed and fabricated considering all these criteria, while having not yet been fully optimized.

Tests of UC-Ika 1 reveal its satisfactory performance during cruising. The swimming efficiency of the robot is mainly investigated using the Strouhal number and the Froude efficiency. UC-Ika 1 swims with the Strouhal number of 0.72 which is not completely ideal value. However, the robot has also very good efficiency in comparison with screw propeller underwater robots and real fishes in nature. UCIka 1 has an efficiency of $78 \%$. This value is obtained while the speed of the robot is comparable with other tuna-like robots 


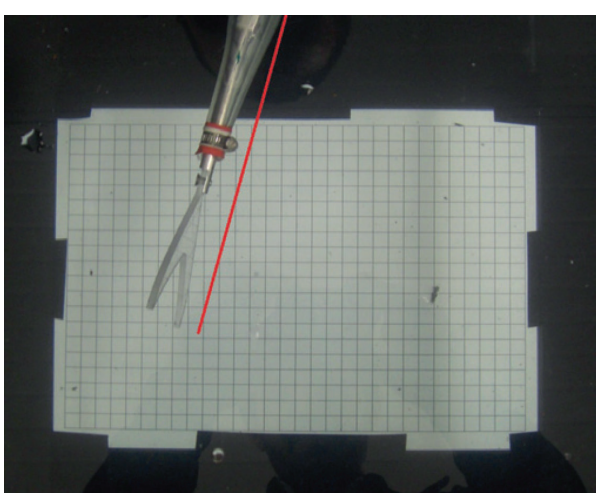

(a) Fixed joint

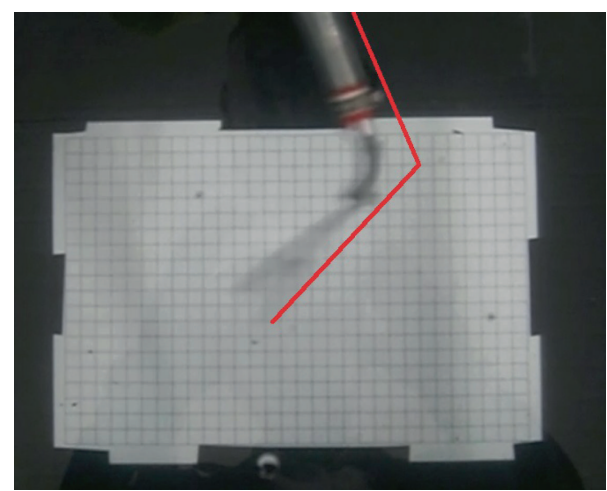

(b) Flexible joint

FIGURE 8: Connections of the caudal fin to the tail peduncle.

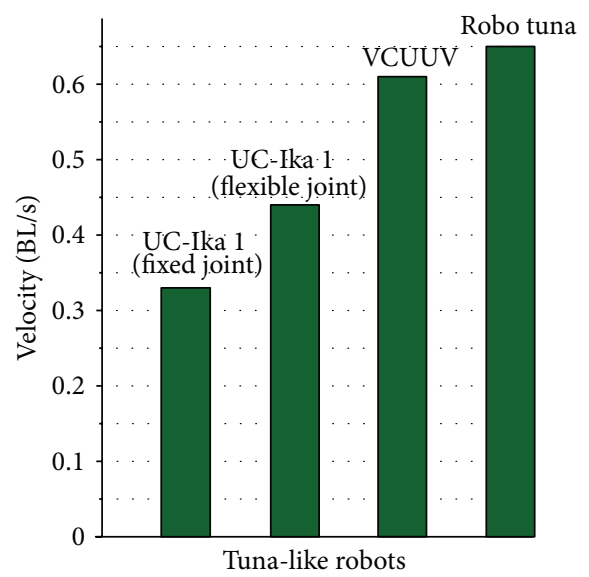

FIGURE 9: Comparison of swimming speed of two tuna-like robots with UC tuna-like robot.

like RoboTuna, which is carriage mounted and uses external power supply, and VCUUV, which swims using hydraulic power unit [9]. The robot reaches a speed of $0.44 \mathrm{BL} / \mathrm{s}$ during cruising with the flexible joint of its caudal fin which is $25 \%$ more than its speed with the fixed joint.

For future prototypes, the efficiency and speed of the robot will be optimized using an optimization algorithm. Additionally, future robot design will include oscillating pectoral fins which add maneuverability capabilities to the robot.

\section{Conflict of Interests}

The authors declare that there is no conflict of interests regarding the publication of this paper.

\section{References}

[1] J. Yu, M. Tan, S. Wang, and E. Chen, "Development of a biomimetic robotic fish and its control algorithm," IEEE Transactions on Systems, Man, and Cybernetics B: Cybernetics, vol. 34, no. 4, pp. 1798-1810, 2004.
[2] H. Hu, J. Liu, I. Dukes, and G. Francis, "Design of 3D swim patterns for autonomous robotic fish," in Proceedings of the IEEE/RSJ International Conference on Intelligent Robots and Systems (IROS '06), pp. 2406-2411, October 2006.

[3] M. S. Triantafyllou and G. S. Triantafyllou, "An efficient swimming machine," Scientific American, vol. 272, no. 3, pp. 40-48, 1995.

[4] J. M. Anderson and N. K. Chhabra, "Maneuvering and stability performance of a robotic tuna," Integrative and Comparative Biology, vol. 42, no. 1, pp. 118-126, 2002.

[5] J. Liu, I. Dukes, R. Knight, and H. Hu, "Development of fishlike swimming behaviours for an autonomous robotic fish," in Proceedings of the Control Conference, vol. 4, 2004.

[6] D. Lachat, A. Crespi, and A. Ijspeert, "Boxybot, the fish robot design and realization," EPFL Semester Project 27, 2005.

[7] P. Kodati, J. Hinkle, A. Winn, and X. Deng, "Microautonomous robotic ostraciiform (MARCO): hydrodynamics, design, and fabrication," IEEE Transactions on Robotics, vol. 24, no. 1, pp. 105-117, 2008.

[8] J. Liang, T. Wang, and L. Wen, "Development of a two-joint robotic fish for real-world exploration," Journal of Field Robotics, vol. 28, no. 1, pp. 70-79, 2011.

[9] S. F. Masoomi, S. Gutschmidt, X. Chen, and M. Sellier, "Novel swimming mechanism for a robotis fish," in Engineering Creative Design in Robotics and Mechatronics, pp. 41-58, IGI Global, Hershey, Pa, USA, 2013.

[10] M. Sfakiotakis, D. M. Lane, and J. B. C. Davies, "Review of fish swimming modes for aquatic locomotion," IEEE Journal of Oceanic Engineering, vol. 24, no. 2, pp. 237-252, 1999.

[11] P. R. Bandyopadhyay, "Trends in biorobotic autonomous undersea vehicles," IEEE Journal of Oceanic Engineering, vol. 30, no. 1, pp. 109-139, 2005.

[12] R. Alexander, Principles of Animal Locomotion, Princeton University Press, Princeton, NJ, USA, 2002.

[13] J. Videler, Fish Swimming, vol. 10, Springer, New York, NY, USA, 1993.

[14] G. S. Triantafyllou, M. S. Triantafyllou, and M. A. Grosenbaugh, "Optimal thrust development in oscillating foils with application to fish propulsion," Journal of Fluids and Structures, vol. 7, no. 2, pp. 205-224, 1993.

[15] M. Lighthill, "Note on the swimming of slender fish," Journal of Fluid Mechanics, vol. 9, no. 2, pp. 305-317, 1960. 
[16] S. Masoomi, S. Gutschmidt, X. Chen, and M. Sellier, "Mathematical modelling and parameter optimization of a 2-dof fish robot," in Proceedings of the 19th International Conference on Mechatronics and Machine Vision in Practice (M2VIP '12), pp. 212-217, 2012.

[17] J. Yu and L. Wang, "Parameter optimization of simplified propulsive model for biomimetic robot fish," in Proceedings of the IEEE International Conference on Robotics and Automation (ICRA '05), pp. 3306-3311, April 2005.

[18] C. K. Chua, K. F. Leong, and C. C. S. Lim, Rapid Prototyping: Principles and Applications, World Scientific Publishing, River Edge, NJ, USA, 2010.

[19] Sylgard 184 silicone elastomer,Brochure, Dow Corning, April 2013, http://www.dowcorning.com/DataFiles/ 090276fe80190b08.pdf.

[20] C. Mavroidis, C. Pfeiffer, and M. Mosley, "5.1 conventional actuators, shape memory alloys, and electrorheological fluids," in Automation, Miniature Robotics and Sensors for Nondestructive Testing and Evaluation, vol. 4, p. 189, 2000. 

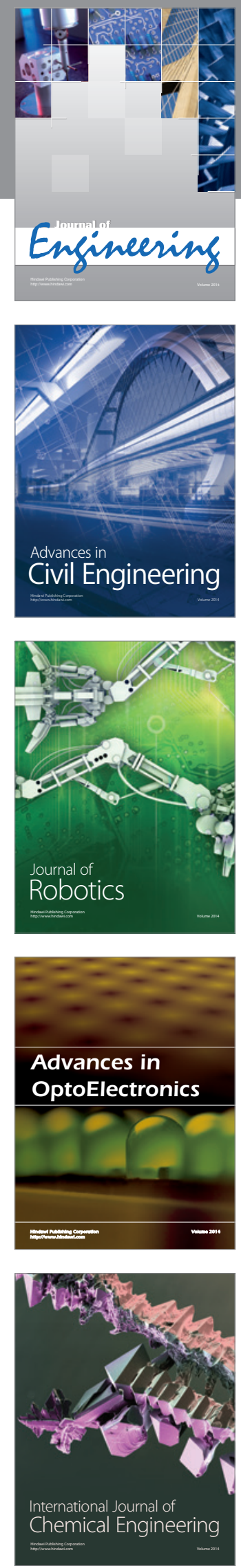

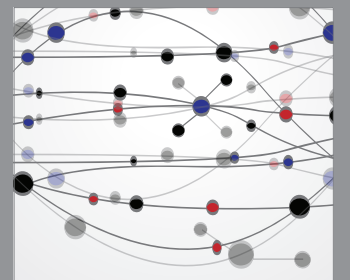

The Scientific World Journal
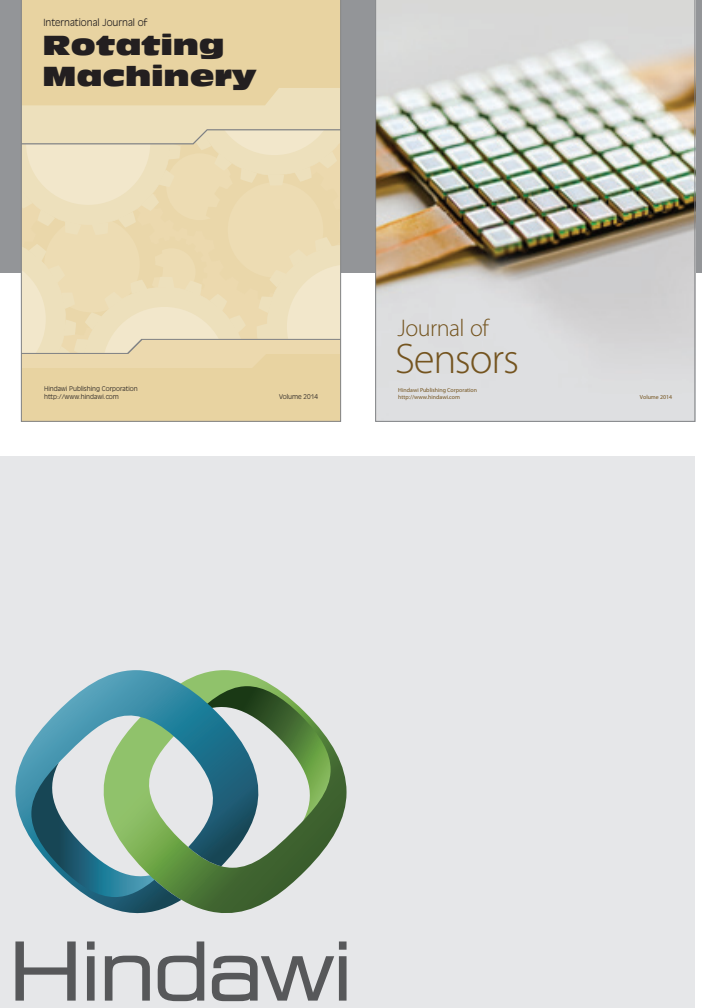

Submit your manuscripts at http://www.hindawi.com
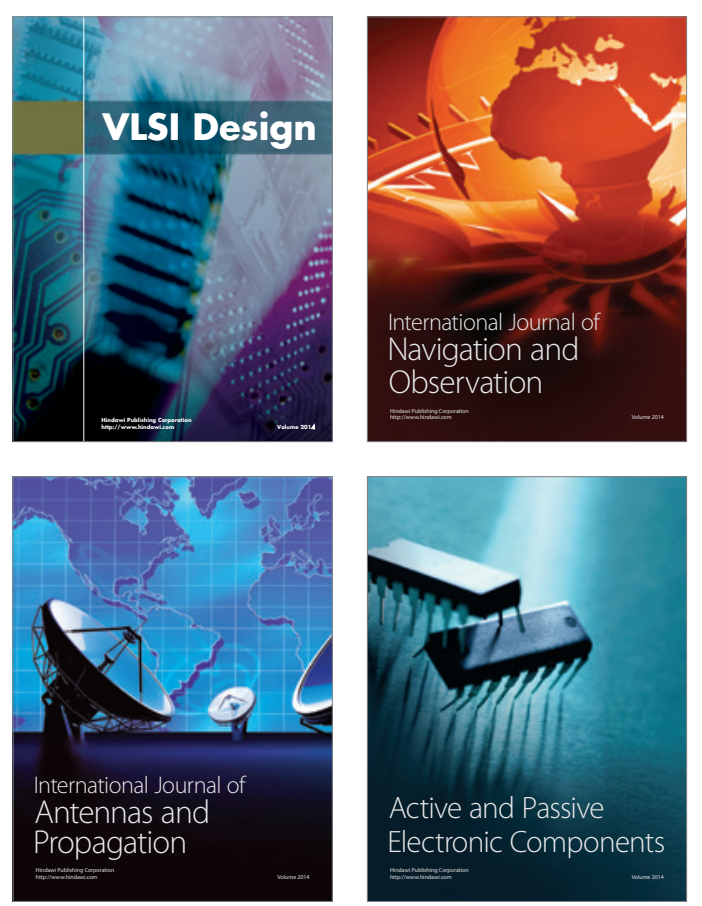
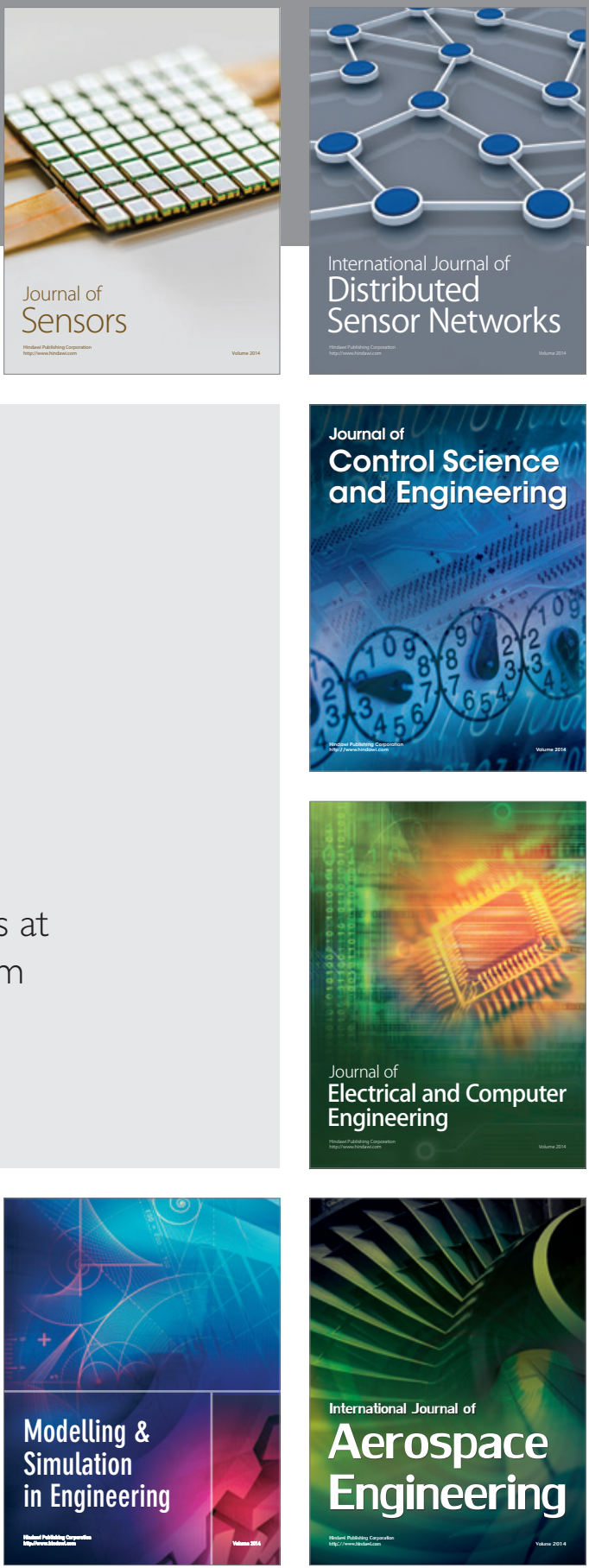

Journal of

Control Science

and Engineering
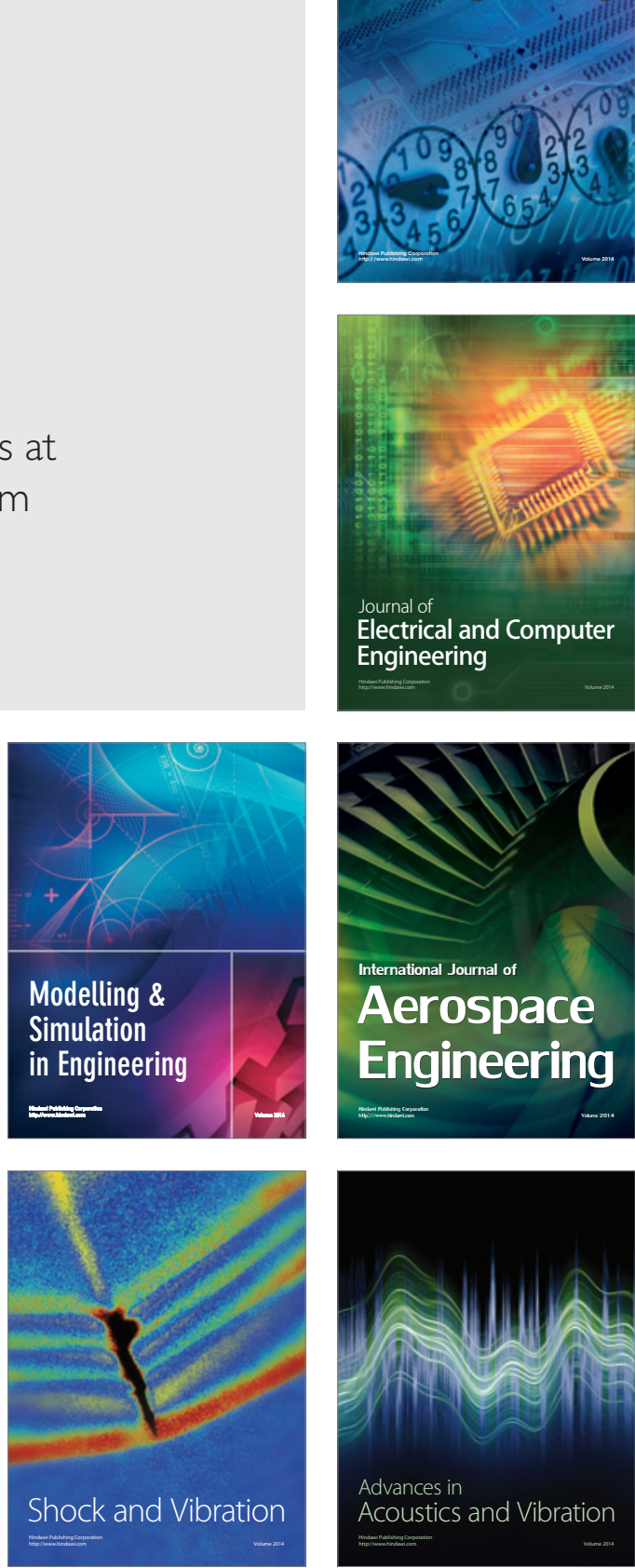